\title{
INFLUÊNCIA DE TRÊS DIFERENTES EMBALAGENS DE PVC NA VIDA PÓS-COLHEITA DE NÊSPERA ${ }^{1}$
}

\author{
ANDERSON ADRIANO MARTINS MELO ${ }^{2}$ \\ LUIZ CARLOS DE OLIVEIRA LIMA ${ }^{3}$
}

\begin{abstract}
RESUMO - Foram pesquisados os efeitos de três diferentes densidades de filme de $\operatorname{PVC}(15,20$ e $30 \mu \mathrm{m})$ na conservação de nêspera armazenada a $3 \pm 2{ }^{\circ} \mathrm{C}$ e 85 $\pm 3 \%$ UR durante 50 dias, sendo, para isso, analisados parâmetros físico-químicos. Houve um decréscimo na acidez titulável em todos os tratamentos durante o armazenamento. Os tratamentos PVC 20 e 30 foram os mais eficientes na redução da perda de massa dos frutos frescos. Os maiores teores dos açúcares totais e sacarose foram obtidos nos tratamentos PVC 20 e 30; porém, nos 20 primeiros dias de armazenamento não houve hi-
\end{abstract}

drólise de sacarose e diminuição significativa da glicose. Observou-se no período de armazenamento um incremento na solubilização de pectinas com uma redução no teor de pectina total. Além disso, o tratamento PVC 30 exibiu os menores teores de pectina solúvel. Houve, durante o armazenamento, uma menor hidrólise de açúcares nos tratamentos PVC 20 e 30 em comparação com outros tratamentos. Porém, o tratamento PVC 20 apresenta-se economicamente como o mais indicado para conservação pós-colheita de nêspera, quando armazenada por um período de até 50 dias.

TERMOS PARA INDEXAÇÃO: Nêspera, pós-colheita, armazenamento, PVC.

\section{INFLUENCE OF THREE DIFFERENT PVC PACKAGES IN THE POSTHARVEST LIFE OF LOQUAT}

\begin{abstract}
The effects of three different densities of PVC film $(15,20$ and $30 \mu \mathrm{m})$ were investigated for loquat conservation during storage at $3 \pm 2{ }^{\circ} \mathrm{C}$ and $85 \pm$ $3 \% \mathrm{RH}$ for fifty days, by physical-chemical parameters analysis. There was a decrease in total titratable acidity, in all treatments during storage. The treatments PVC 20 and 30 were the most efficient for reduction of fresh fruit mass losses. The highest total sugar and sucrose contents were obtained in treatments PVC 20 and 30. However, during the early days of storage, there was no
\end{abstract}

INDEX TERMS: Loquat, postharvest, storage, PVC.

\section{INTRODUÇÃO}

A nespereira (Eriobotrya japonica Lindl.), pertencente à família rosaceae e subfamília Prunoideae (PENTEADO, 1986), é originária do sudoeste da China, porém, só se tornou conhecida mundialmente quando foi introduzida por imigrantes chineses no Japão onde teve uma grande aceitação, passando a ser cultivada nesse país por mais de 1000 anos. O Japão é hydrolysis of sucrose and neither significant decrease of glucose. During storage, it was observed an increase in pectin solubility with a reduction in the total pectin. Furthermore, the PVC30 treatment exhibited the smaller soluble pectin contents. There was, during the storage, a smaller sugar hydrolysis in PVC 20 and 30, in comparison with another treatments. However the PVC 20 treatment economically showed to be the most indicated for post-harvest conservation of loquat when used for storage in a period of 50 days.

considerado o maior produtor de nêspera "in natura", seguido por Israel e Brasil (LOQUAT, 1997). No Brasil, segundo Penteado (1986), o Estado de São Paulo é o maior produtor dessa fruta, concentrando a produção principalmente em Mogi das Cruzes e São Miguel Arcanjo. As cultivares mais adaptadas e produzidas no Brasil são, principalmente, Mizuho (cruzamento das cultivares Kuzunoki com a Tanaka) e Mogi, considerada a primeira cultivar comercial, e a mais estudada.

1. Projeto financiado pelo CNPq.

2. Aluno de Graduação do curso de Agronomia da UNIVERSIDADE FEDERAL DE LAVRAS/UFLA - Caixa Postal 37 - 37200-000 - Lavras, MG. Estudante de Iniciação Cientifica do Departamento de Ciência dos Alimentos.

3. Professor Adjunto do DCA/UFLA. 
O uso de atmosfera modificada é hoje uma realidade no armazenamento de frutos e hortaliças, sendo um forte complemento para se manter a qualidade e estender a vida pós-colheita, desde que se tenha o controle do binômio temperatura e umidade. A atmosfera modificada baseia-se na combinação das características de permeabilidade do filme utilizado com a taxa de respiração e produção de etileno dos frutos. Havendo essa correta combinação, propicia-se uma atmosfera apropriada dentro da embalagem para um favorável consumo de $\mathrm{O}_{2}$ e produção de $\mathrm{CO}_{2}$ pela respiração. Entre os filmes mais utilizados, estão os de polietileno de baixa densidade e os PVC (cloretos de polivinila) (KADER, 1998).

Os frutos têm diferentes limites de tolerância aos níveis reduzidos de $\mathrm{O}_{2}$ e elevados de $\mathrm{CO}_{2}$. A redução dos níveis de $\mathrm{O}_{2}$ a valores abaixo de $2 \%$ pode conduzir à respiração anaeróbica, enquanto o acúmulo excessivo de $\mathrm{CO}_{2}$ também é deletério (MOLEYAR e NARASIMHAM, 1994). Dessa forma, a seleção de um filme com permeabilidade compatível à taxa de respiração do produto e o controle da temperatura são requisitos importantes para o armazenamento em atmosfera modificada. Flutuações na temperatura de armazenamento, aumento na taxa respiratória e impermeabilidade do filme podem alterar o equilíbrio de gases dentro da embalagem, conduzindo a condições inadequadas de armazenamento (PÉREZ et al., 1997).

Para se conseguir uma atmosfera satisfatória, a permeabilidade do filme selecionado deve permitir a entrada de $\mathrm{O}_{2}$ e a saída de $\mathrm{CO}_{2}$ numa taxa equivalente ao consumo de $\mathrm{O}_{2}$ e à produção de $\mathrm{CO}_{2}$ pelo produto. Em geral, pretende-se que a permeabilidade ao $\mathrm{CO}_{2}$ seja de três a cinco vezes maior que a permeabilidade ao $\mathrm{O}_{2}$. As embalagens mais utilizadas no armazenamento de frutos e hortaliças são de polietileno de baixa densidade e de cloreto de polivinila, usados como filme envoltório. O polietileno (PE) é utilizado como saco para acondicionamento de frutos minimamente processados, tendo em vista que atendem bem a esse requisito (ZAGORY e KADER, 1988).

O transporte de nêsperas seja feito em atmosfera modificada e as mesmas sejam mantidas a baixas temperaturas, diminuindo a sua taxa respiratória e evitando danos mecânicos comuns no transporte a longas distâncias.

Segundo Flores e Rívas (1975) e Ding et al. (1998a, 1998b), a faixa ideal de temperatura para o armazenamento de nêsperas varia entre $1^{\circ} \mathrm{C}$ e $5^{\circ} \mathrm{C}$, po- dendo chegar até a 30 dias, sem comprometimento da qualidade. Temperaturas de armazenamento mais elevadas, iguais a 10 e a $20^{\circ} \mathrm{C}$, propiciam vida útil de 15 e 20 dias, respectivamente.

Com o presente trabalho objetivou-se avaliar a influência de três espessuras de filme de PVC e cinco períodos distintos de armazenamento sobre o comportamento bioquímico e fisiológico de nêsperas cv. Mogi.

\section{MATERIAL E MÉTODOS}

Os frutos utilizados foram colhidos na fazenda Ogawa, município de Mogi das Cruzes, São Paulo, no estádio de maturação 6 (coloração da casca amareloclara para escura) segundo valores de cores de Hunter, descrito por Hamauzu et al. (1997), com peso médio de 40 g e transportados para o Laboratório de Fisiologia Pós-colheita de Frutos e Hortaliças (DCA-UFLA). Os frutos foram mergulhados em solução de hipoclorito de sódio a $0,1 \%$ por um minuto para desinfecção. Após a imersão, os frutos ficaram expostos à temperatura ambiente para eliminar o excesso de umidade e foram acondicionados em bandejas plásticas contendo 10 frutos, sendo essas embaladas, com exceção do controle, com filmes de polietileno de três densidades: 15,20 e $30 \mu \mathrm{m}$.

Em seguida, foram armazenados em câmara fria a $3 \pm 2^{\circ} \mathrm{C}$ e $85 \pm 3 \%$ UR, por 50 dias. As análises físicas (perda de massa), físico-químicas (acidez total titulável) e bioquímicas (substâncias pécticas e açúcares totais e redutores) foram realizadas no tempo zero $(0$ dias) e a cada 10 dias.

As perdas de massa foram obtidas por gravimetria, mediante diferença entre a massa inicial e aquela obtida nos frutos da parcela experimental, a cada intervalo de amostragem, com o auxílio de uma balança semi-analítica, sendo os resultados expressos em $\mathrm{g} / 100 \mathrm{~g}$

A acidez total titulável (ATT) foi determinada por titulometria com solução de $\mathrm{NaOH} 0,1 \mathrm{~N}$, de acordo com INSTITUTO ADOLFO LUTZ (1985), e expressa em g/100 g de ácido málico.

Os Açúcares totais e redutores foram determinados pelo método redutométrico de Somogyi, adaptado por Nelson (1944). Os resultados de glicose e sacarose foram expressos em $\mathrm{g} / 100 \mathrm{~g}$ de polpa fresca.

As pectinas total e solúvel foram extraídas segundo técnica de McCready e McComb (1952), e analisadas pela técnica modificada do Carbazol (BITTER e 
MUIR, 1962). Os resultados foram expressos em $\mathrm{mg}$ de ácido galacturônico por $100 \mathrm{~g}$ de polpa fresca.

$\mathrm{O}$ experimento foi realizado em delineamento inteiramente casualizado em esquema fatorial duplo (4 tratamentos e 3 repetições) e 6 períodos de amostragem. Cada parcela experimental foi constituída por 10 frutos. Os resultados foram submetidos à análise de variância e as médias, comparadas pelo teste de Tukey, a $5 \%$ de probabilidade.

\section{RESULTADOS E DISCUSSÃO}

Não houve influência significativa da interação embalagem e período de armazenamento no teor de acidez total titulável (ATT). Entretanto, foram observadas diferenças significativas nos fatores isoladamente. Aos 30 e 50 dias de armazenamento, houve diferença significativa entre as embalagens de 15 e $20 \mu \mathrm{m}$ e 20 e $30 \mu \mathrm{m}$, respectivamente (Tabela 1). Em ambos os casos, os frutos embalados em filmes de PVC $20 \mu \mathrm{m}$ apresentaram as menores médias de ATT, enquanto os demais tratamentos, mesmo apresentando médias maiores que o controle, não diferiram estatisticamente. No entanto, a maior média foi observada para o tratamento de frutos em embalagens de PVC 30 $\mu \mathrm{m}$. A partir do trigésimo dia de armazenamento houve aumento de ATT, ocorrendo uma maior utilização dos ácidos totais tituláveis a partir do quadragésimo dia. Em todos os tratamentos, com exceção do PVC $20 \mu \mathrm{m}$, não houve diferença significativa no teor de ATT, com o tempo de armazenamento. Esse comportamento assemelha-se ao encontrado por Ding et al. (1998a) e Chachin e Hamauzu (1997), que observaram uma rápida diminuição na concentração dos ácidos málico e fumárico.

Houve decréscimo na ATT no decorrer do período de armazenamento (Figura 1). Segundo Chitarra e Chitarra (1990), o teor de ácidos orgânicos, com poucas exceções, diminui com a maturação, em decorrência do processo respiratório ou de sua conversão em açúcares, já que constituem excelentes reservas energéticas em frutos, por sua oxidação no ciclo de Krebs. Ding et al. (1997a) observaram o mesmo comportamento em nêsperas.

Os tratamentos com filme de PVC reduziram a perda de massa durante o período de armazenamento, e PVC 20 e $30 \mu \mathrm{m}$ foram mais eficientes (Tabela 2), diferindo estatisticamente entre si nos 50 dias de armazenamento, quando o PVC 30 apresentou a menor média $(1,29 \mathrm{~g} / 100 \mathrm{~g})$, enquanto PVC 20 obteve a média de 1,65 g/100 g. Isso se justifica pelo fato de o PVC 30 ser mais espesso que PVC 20, contribuindo para que haja, além de uma melhor troca de gases, uma melhor conservação da umidade no interior da embalagem, acarretando, com isso, uma menor perda de massa. A perda média de massa no tratamento PVC $20 \mu \mathrm{m}$ foi de $0,79 \%$ e no tratamento PVC $30 \mu \mathrm{m}$, de $0,72 \%$, quando comparados às suas massas iniciais, enquanto o controle teve uma perda de $6,22 \%$ (Tabela 2). O uso da atmosfera modificada não influenciou na qualidade de apresentação dos frutos, com relação à sua aparência e turgidez.

Os filmes de densidades mais elevadas são mais eficientes na redução da perda de massa e na manutenção da aparência.

TABELA 1 - Valores médios de acidez titulável total de nêsperas 'Mogi' acondicionadas em filmes de PVC de diferentes espessuras e armazenadas a $3 \pm 2{ }^{\circ} \mathrm{C}$ e $85 \pm 3 \% \mathrm{UR}$, por 50 dias.

\begin{tabular}{cccccccc}
\hline \multicolumn{7}{c}{ ATT (g/100 g de ácido málico) durante armazenamento (dias) } \\
\hline Trat. & $\mathbf{0}$ & $\mathbf{1 0}$ & $\mathbf{2 0}$ & $\mathbf{3 0}$ & $\mathbf{4 0}$ & $\mathbf{5 0}$ & Média \\
\hline CONT. & $0,59 \mathrm{a}$ & $0,31 \mathrm{a}$ & $0,28 \mathrm{a}$ & $0,24 \mathrm{ab}$ & $0,19 \mathrm{a}$ & $0,20 \mathrm{ab}$ & $0,30 \mathrm{ab}$ \\
PVC15 & $0,59 \mathrm{a}$ & $0,37 \mathrm{a}$ & $0,36 \mathrm{a}$ & $0,32 \mathrm{a}$ & $0,18 \mathrm{a}$ & $0,21 \mathrm{ab}$ & $0,33 \mathrm{ab}$ \\
PVC20 & $0,59 \mathrm{a}$ & $0,27 \mathrm{a}$ & $0,37 \mathrm{a}$ & $0,21 \mathrm{~b}$ & $0,22 \mathrm{a}$ & $0,13 \mathrm{~b}$ & $0,30 \mathrm{~b}$ \\
PVC30 & $0,59 \mathrm{a}$ & $0,32 \mathrm{a}$ & $0,32 \mathrm{a}$ & $0,27 \mathrm{ab}$ & $0,28 \mathrm{a}$ & $0,26 \mathrm{a}$ & $0,34 \mathrm{a}$ \\
\hline
\end{tabular}

${ }^{(1)}$ Médias seguidas de mesmas letras, minúsculas na vertical não diferem entre si pelo teste de Tukey a $\mathrm{P}<$ $\mathbf{0 , 0 5 \%}$.

Ciênc. agrotec., Lavras. V.27, n.6, p.1330-1339, nov./dez., 2003 


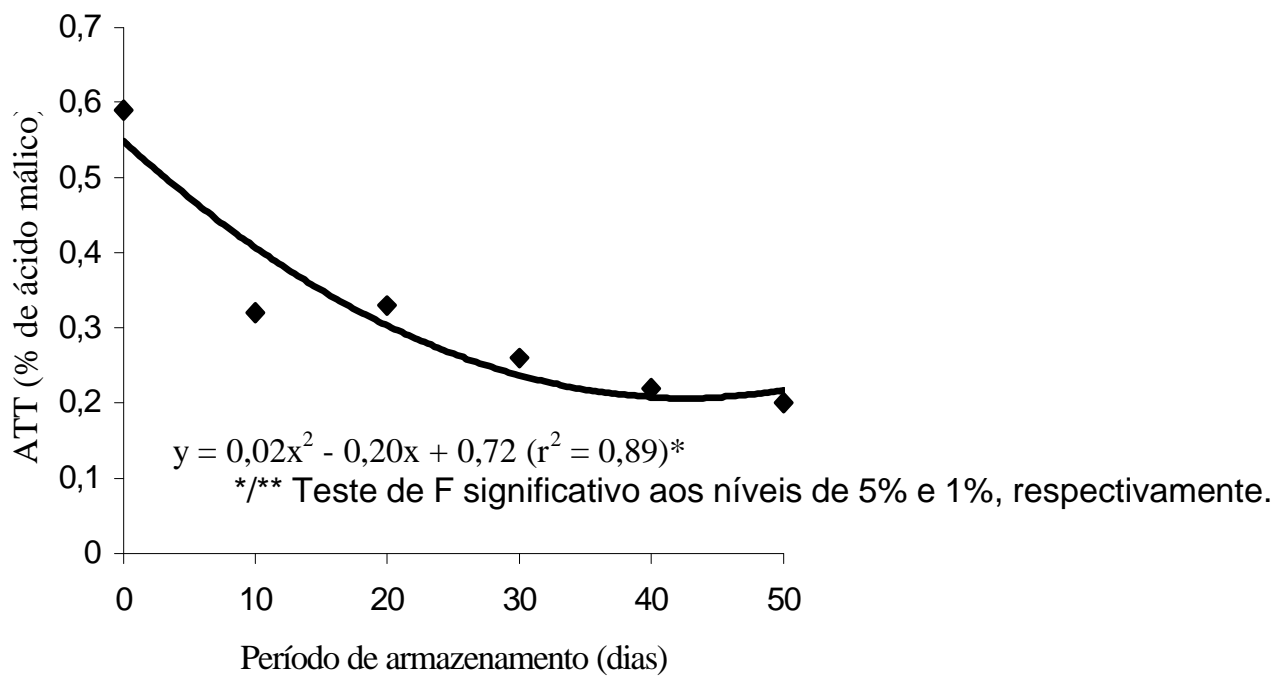

FIGURA 1 - Curva e equação de regressão representativa do valor de acidez total titulável de nêsperas 'Mogi' acondicionadas em filme de PVC de diferentes espessuras e armazenadas a $3 \pm 2^{\circ} \mathrm{C}$ e $85 \pm 3 \%$ UR, por 50 dias.

TABELA 2 - Valores médios referentes à perda de massa de nêsperas 'Mogi' acondicionadas em filmes de PVC de diferentes espessuras e armazenadas a $3 \pm 2{ }^{\circ} \mathrm{C}$ e $85 \pm 3 \%$ UR, por 50 dias.

\begin{tabular}{cccccccc}
\hline \multicolumn{7}{c}{ Perda de massa fresca $(\mathbf{g} / \mathbf{1 0 0}$ g) } & durante o período de armazenamento (dias) \\
\hline Trat. & $\mathbf{0}$ & $\mathbf{1 0}$ & $\mathbf{2 0}$ & $\mathbf{3 0}$ & $\mathbf{4 0}$ & $\mathbf{5 0}$ & Média \\
\hline CONT. & 0,00 & $3,24 \mathrm{a}$ & $4,26 \mathrm{a}$ & $7,46 \mathrm{a}$ & $10,42 \mathrm{a}$ & $11,92 \mathrm{a}$ & $6,22 \mathrm{a}$ \\
PVC 15 & 0,00 & $0,71 \mathrm{~b}$ & $0,95 \mathrm{~b}$ & $1,84 \mathrm{~b}$ & $1,60 \mathrm{~b}$ & $2,34 \mathrm{~b}$ & $1,24 \mathrm{~b}$ \\
PVC 20 & 0,00 & $0,45 \mathrm{c}$ & $0,54 \mathrm{c}$ & $0,95 \mathrm{c}$ & $1,15 \mathrm{c}$ & $1,65 \mathrm{c}$ & $0,79 \mathrm{c}$ \\
PVC 30 & 0,00 & $0,37 \mathrm{c}$ & $0,61 \mathrm{c}$ & $0,98 \mathrm{c}$ & $1,09 \mathrm{c}$ & $1,29 \mathrm{~d}$ & $0,72 \mathrm{c}$ \\
\hline
\end{tabular}

${ }^{(1)}$ Médias seguidas de mesma letra na coluna não diferem entre si pelo teste de Tukey a $P<0,05$.

Houve efeito significativo da embalagem, período de armazenamento e interação embalagem x período sobre os teores de açúcares totais (AT), redutores e nãoredutores. Observou-se uma superioridade nos valores de AT nos frutos do tratamento PVC 30 mais significativa no trigésimo e quadragésimo dia de armazenamento (Tabela 3).

O tratamento PVC $30 \mu \mathrm{m}$ apresentou-se como o mais eficiente na manutenção do teor de AT, enquanto os frutos do tratamento PVC $15 \mu \mathrm{m}$ não diferiram do controle, sendo esse significativo em todos os períodos de armazenamento, com menor média geral (Tabela 3).
Os teores de AT nos tratamentos PVC 15, 20 e $30 \mu \mathrm{m}$ aumentaram até os 40 dias de armazenamento, decrescendo logo em seguida até os 50 dias (Figura 2). Os resultados obtidos estão de acordo com os encontrados em nêsperas por Ding et al. (1997a, 1997b) e Ding et al. (1998b), que também observaram pouca mudança no teor de açúcares totais nos primeiros 30 dias e uma diminuição após 60 dias de armazenamento, o que pode ser conseqüência da hidrólise de sacarose em glucose e frutose, provavelmente pela sua utilização nas reações catabólicas que ocorrem no início da senescência dos frutos. 


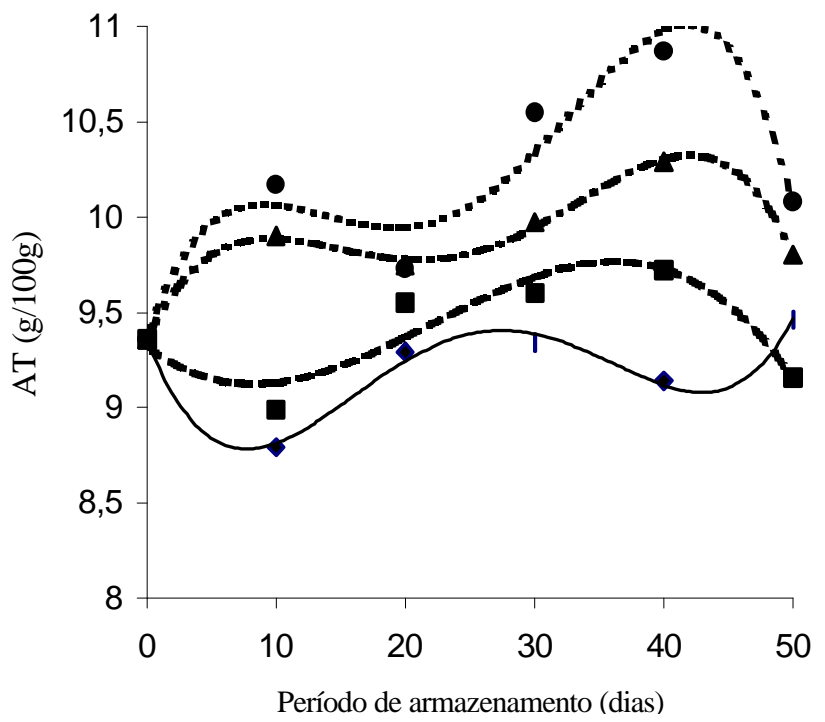

$$
\begin{aligned}
\bullet & \text { CONTROLE } \\
y= & 0,05 x^{4}-0,69 x^{3}+3,42 x^{2}-6,71 x+13,28\left(r^{2}=0,98\right)^{* *}(\text { Controle }) \\
y= & -0,059 x^{3}+0,57 x^{2}-1,48 x+10,29\left(\mathrm{r}^{2}=0,84\right)^{* *}(\text { PVC } 15) \\
y= & -0,041 x^{4}+0,56 x^{3}-2,66 x^{2}+5,19 x+6,32\left(r^{2}=0,99\right)^{* *}(\text { PVC } 20) \\
y= & -0,065 x^{4}+0,87 x^{3}-3,98 x^{2}+7,52 x+5,037\left(r^{2}=0,92\right)^{* *}(\text { PVC } 30) \\
& * * * \text { Teste de F significativo aos níveis de } 5 \% \text { e } 1 \%, \text { respectivamente. }
\end{aligned}
$$

FIGURA 2 - Curva e equações de regressão representativas dos valores de açúcares totais (AT) de nêsperas 'Mogi' acondicionadas em filme de PVC de diferentes espessuras e armazenadas a $3 \pm 2{ }^{\circ} \mathrm{C}$ e $85 \pm 3 \%$ UR, por 50 dias.

TABELA 3 - Valores médios de açúcares totais (AT) de nêsperas 'Mogi' acondicionadas em filmes de PVC de diferentes espessuras e armazenadas a $3 \pm 2^{\circ} \mathrm{C}$ e $85 \pm 3 \% \mathrm{UR}$, por 50 dias.

\begin{tabular}{cccccccc}
\hline \multicolumn{7}{c}{ AT (g/ 100 g) durante o período de armazenamento (dias) } \\
\hline Trat. & $\mathbf{0}$ & $\mathbf{1 0}$ & $\mathbf{2 0}$ & $\mathbf{3 0}$ & $\mathbf{4 0}$ & $\mathbf{5 0}$ & Média \\
\hline CONT. & $9,36 \mathrm{a}$ & $8,79 \mathrm{~b}$ & $9,29 \mathrm{a}$ & $9,34 \mathrm{c}$ & $9,14 \mathrm{~d}$ & $9,46 \mathrm{bc}$ & $9,23 \mathrm{c}$ \\
PVC 15 & $9,36 \mathrm{a}$ & $8,99 \mathrm{~b}$ & $9,55 \mathrm{a}$ & $9,60 \mathrm{bc}$ & $9,72 \mathrm{c}$ & $9,16 \mathrm{c}$ & $9,40 \mathrm{c}$ \\
PVC 20 & $9,36 \mathrm{a}$ & $9,90 \mathrm{a}$ & $9,75 \mathrm{a}$ & $9,97 \mathrm{~b}$ & $10,29 \mathrm{~b}$ & $9,80 \mathrm{ab}$ & $9,85 \mathrm{~b}$ \\
PVC 30 & $9,36 \mathrm{a}$ & $10,17 \mathrm{a}$ & $9,73 \mathrm{a}$ & $10,55 \mathrm{a}$ & $10,87 \mathrm{a}$ & $10,08 \mathrm{a}$ & $10,13 \mathrm{a}$ \\
\hline
\end{tabular}

\footnotetext{
${ }^{(1)}$ Médias seguidas de mesma letra na coluna não diferem entre si pelo teste de Tukey a $P<0,05$.
}

Os frutos dos tratamentos PVC 20 e 30 foram estatisticamente iguais em todos os tempos de armazenamento e apresentaram teores mais baixos de açúcares redutores (Tabela 4), sendo o PVC 30 significativamente diferente dos valores médios apresentados pelos frutos dos tratamen- tos PVC 15 e controle, o que vale salientar que esse resultado indica maior eficiência dos tratamentos que envolvem filmes de PVC 20 e $30 \mu \mathrm{m}$ no retardamento da senescência de nêspera. $\mathrm{O}$ teor de açúcares redutores decresceu em todos os tratamentos, nos 20 primeiros dias de armazena- 
mento, menos no controle, no qual não foi observada variação significativa (Tabela 4), divergindo do comportamento de elevação observado por Ding et al. (1998a, 1998b, 1998c). Pode-se inferir que nos frutos sob atmosfera modificada houve, no início do armazenamento, aumento das reações metabólicas, com utilização dos açúcares redutores no processo respiratório, com vista à produção da energia.

Os frutos do tratamento PVC $30 \mu \mathrm{m}$ apresentaram as maiores médias no teor de sacarose, seguidas pelo PVC 20 (Tabela 5), o que indica a influência do uso da atmosfera modificada na redução da hidrólise de sacarose. Observou-se um decréscimo no teor de sacarose em todos os tratamentos envolvendo filmes de PVC (Figura 3), enquanto os frutos do tratamentocontrole não apresentaram alterações significativas. Também se torna evidente uma maior concentração na sacarose nos 20 primeiros dias de armazenamento, decrescendo logo em seguida até os 50 dias. Os resultados obtidos divergem, em parte, dos encontrados por Ding et al. (1997a, 1997b) e Ding et al. (1998b, 1998c), segundo os quais os teores de sacarose decresceram rapidamente no decorrer do armazenamento.

TABELA 4 - Valores médios de açúcares redutores (glucose) de nêsperas 'Mogi' acondicionadas em filmes de PVC de diferentes espessuras e armazenadas a $3 \pm 2{ }^{\circ} \mathrm{C}$ e $85 \pm 3 \%$ UR, por 50 dias.

\begin{tabular}{cccccccc}
\hline \multicolumn{7}{c}{ Açúcares redutores $(\mathbf{g} / \mathbf{1 0 0} \mathbf{g})$ durante o período de armazenamento (dias) } \\
\hline Trat. & $\mathbf{0}$ & $\mathbf{1 0}$ & $\mathbf{2 0}$ & $\mathbf{3 0}$ & $\mathbf{4 0}$ & $\mathbf{5 0}$ & Médias \\
\hline CONT. & $7,43 \mathrm{a}$ & $7,31 \mathrm{a}$ & $7,11 \mathrm{a}$ & $7,96 \mathrm{a}$ & $7,29 \mathrm{~b}$ & $7,44 \mathrm{~b}$ & $7,42 \mathrm{ab}$ \\
PVC 15 & $7,43 \mathrm{a}$ & $6,92 \mathrm{ab}$ & $7,11 \mathrm{a}$ & $7,37 \mathrm{~b}$ & $8,26 \mathrm{a}$ & $8,13 \mathrm{a}$ & $7,54 \mathrm{a}$ \\
PVC 20 & $7,43 \mathrm{a}$ & $6,83 \mathrm{~b}$ & $6,65 \mathrm{~b}$ & $7,12 \mathrm{~b}$ & $7,61 \mathrm{~b}$ & $7,86 \mathrm{ab}$ & $7,25 \mathrm{bc}$ \\
PVC 30 & $7,43 \mathrm{a}$ & $6,79 \mathrm{~b}$ & $6,62 \mathrm{~b}$ & $7,44 \mathrm{~b}$ & $7,53 \mathrm{~b}$ & $7,42 \mathrm{~b}$ & $7,21 \mathrm{c}$ \\
\hline
\end{tabular}

${ }^{(1)}$ Médias seguidas de mesma letra na coluna não diferem entre si pelo teste de Tukey a $\mathbf{P}<0,05$.

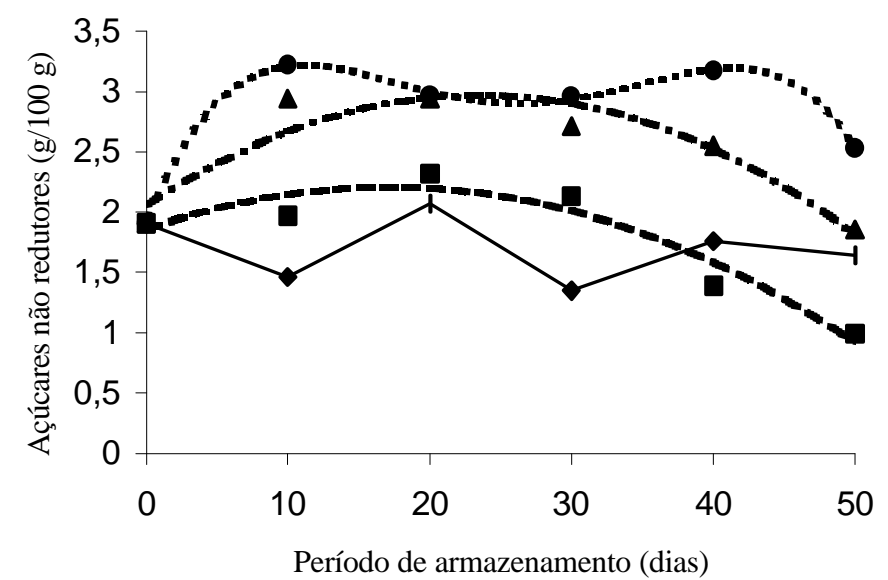

$$
\begin{aligned}
& \text { 千 CONTROLE - - PVC 15-.. - -PVC 20........PVC } 30 \\
& y=-0,12 x^{2}+0,65 x+1,33\left(r^{2}=0,92\right) * *(\text { PVC 15) } \\
& \mathrm{y}=-0,17 \mathrm{x}^{2}+1,11 \mathrm{x}+1,11\left(\mathrm{r}^{2}=0,89\right) * *(\text { PVC } 20) \\
& y=-0,06 x^{4}+0,87 x^{3}-4,47 x^{2}+9,51 x-3,94\left(r^{2}=0,99\right) * *(P V C 30) \\
& { }^{*} /{ }^{* *} \text { Teste de } \mathrm{F} \text { significativo aos níveis de } 5 \% \text { e } 1 \% \text {, respectivamente. }
\end{aligned}
$$

FIGURA 3 - Curvas e equações de regressão representativas dos valores de açúcares não-redutores (sacarose) de nêsperas 'Mogi' acondicionadas em filme de PVC de diferentes espessuras e armazenadas a $3 \pm 2{ }^{\circ} \mathrm{C}$ e $85 \pm 3 \%$ UR, por 50 dias. 
TABELA 5 - Valores médios de açúcares não-redutores (sacarose) de nêsperas 'Mogi' acondicionadas em filmes de PVC de diferentes espessuras e armazenadas a $3 \pm 2{ }^{\circ} \mathrm{C}$ e $85 \pm 3 \%$ UR, por 50 dias.

\begin{tabular}{cccccccc}
\hline \multicolumn{7}{c}{ Açúcares não-redutores (g/ 100 g) } \\
Trat. & $\mathbf{0}$ & $\mathbf{1 0}$ & $\mathbf{2 0}$ & $\mathbf{3 0}$ & $\mathbf{4 0}$ & $\mathbf{5 0}$ & Média \\
\hline CONT. & $1,91 \mathrm{a}$ & $1,46 \mathrm{~b}$ & $2,07 \mathrm{~b}$ & $1,35 \mathrm{c}$ & $1,76 \mathrm{c}$ & $1,64 \mathrm{~b}$ & $1,70 \mathrm{c}$ \\
PVC 15 & $1,91 \mathrm{a}$ & $1,97 \mathrm{~b}$ & $2,32 \mathrm{~b}$ & $2,13 \mathrm{~b}$ & $1,39 \mathrm{c}$ & $0,99 \mathrm{c}$ & $1,78 \mathrm{c}$ \\
PVC 20 & $1,91 \mathrm{a}$ & $2,93 \mathrm{a}$ & $2,94 \mathrm{a}$ & $2,71 \mathrm{ab}$ & $2,55 \mathrm{~b}$ & $1,86 \mathrm{~b}$ & $2,48 \mathrm{~b}$ \\
PVC 30 & $1,91 \mathrm{a}$ & $3,22 \mathrm{a}$ & $2,97 \mathrm{a}$ & $2,96 \mathrm{a}$ & $3,17 \mathrm{a}$ & $2,53 \mathrm{a}$ & $2,79 \mathrm{a}$ \\
\hline
\end{tabular}

${ }^{(1)}$ Médias seguidas de mesma letra na coluna não diferem entre si pelo teste de Tukey a $P<0,05$.

Ao se relacionar o teor de sacarose com os resultados obtidos com os açúcares redutores, verificase que houve uma influência dos tratamentos nos 20 primeiros dias, mais representativa nos tratamentos PVC 20 e $30 \mu \mathrm{m}$, ou seja, praticamente não houve hidrólise de sacarose nesse período. Apesar disso, os tratamentos não alteraram o processo metabólico normal dos frutos, sendo isso evidenciado pela diminuição do conteúdo de glucose nos 20 primeiros dias. Após os 20 dias de armazenamento, pode ter havido uma escassez, por parte do fruto, em matéria-prima para produção de energia (glucose). Portanto, houve hidrólise de sacarose em glucose e frutose, observando-se, assim, uma significativa diminuição no conteúdo de sacarose após os 20 primeiros dias de armazenamento (Figura 5), com exceção dos frutos-controle.

Detectaram-se efeitos significativos da embalagem, período de armazenamento e interação embalagem $\mathrm{x}$ período sobre o teor das pectinas total e solúvel. Pode-se verificar que a concentração de pectina solúvel (P.S) aumentou no decorrer do período de armazenamento (Figura 4). Lima et al. (1996), trabalhando com armazenamento de mangas embaladas com filmes de PVC, também observaram um aumento nos teores de P.S com o avanço do armazenamento. O mesmo foi observado em banana 'Prata' por Vilas Boas (1995) e em goiaba por El-Buluk et al. (1995). O tratamento PVC $30 \mu \mathrm{m}$ apresentou a menor média de concentração de pectina solúvel. Já os tratamentos PVC 15 e 20 não diferenciaram signifi- cativamente entre si, porém, as concentrações de pectina foram menores que a dos frutos-controle.

Quanto ao teor de pectina total (P.T), observou-se efeito significativo nos tratamentos embalagem, tempo de armazenamento e interação embalagem $x$ tempo, conforme Tabela 6. Considerando-se todo o período de armazenamento, pode-se verificar que houve redução no teor de pectina total apenas nos frutos dos tratamentos PVC 20 e 30. Nos frutos dos demais tratamentos, o teor de pectina total retornou aos valores detectados no início do armazenamento, apesar de nos 20 primeiros dias, ter sido observado um decréscimo acentuado no teor de P.T. Esse mesmo comportamento foi observado por Vilas Boas (1995) em banana 'Prata', o qual atribui a redução momentânea no teor de pectina total ao consumo de pectinas como substrato, no momento de maior demanda energética. Os filmes PVC 20 e PVC 30 interferiram no teor de pectina total apenas nos últimos períodos de avaliação, quando se verificaram teores mais baixos desses componentes em comparação com os tratamentos-controle e PVC 15, que apresentaram teores semelhantes durante todo o período experimental (Tabela 6). Os valores médios observados variaram entre 489,68 e 563,48 mg de ácido galacturônico . $100 \mathrm{~g}^{-1}$, semelhante à faixa citada por Femenia et al. (1998).

Pelos resultados obtidos, infere-se que o teor de pectina pode, em geral, permanecer inalterado, ou diminuir, ou mesmo aumentar durante o amadurecimento. 


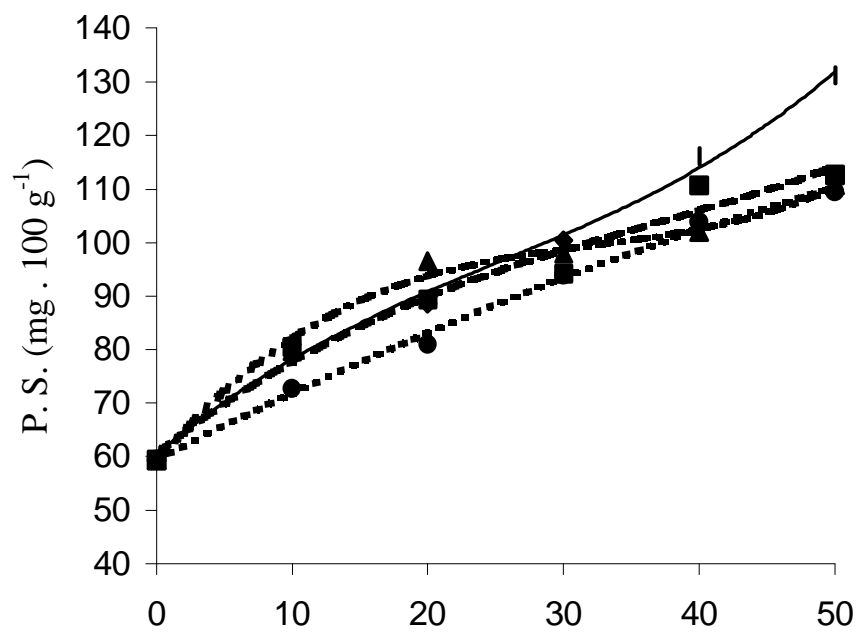

Período de armazenamento (dias)

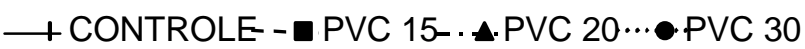

$\mathrm{y}=0,61 \mathrm{x}^{3}-6,42 \mathrm{x}^{2}+33,01 \mathrm{x}+33,03\left(\mathrm{r}^{2}=0,99\right) * *($ Controle $)$

$\mathrm{y}=0,33 \mathrm{x}^{3}-4,61 \mathrm{x}^{2}+28,85 \mathrm{x}+35,85\left(\mathrm{r}^{2}=0,97\right) * *(\mathrm{PVC} 15)$

$\mathrm{y}=0,89 \mathrm{x}^{3}-11,31 \mathrm{x}^{2}+51,14 \mathrm{x}+17,94\left(\mathrm{r}^{2}=0,99\right) * *($ PVC 20$)$

$\mathrm{y}=-0,57 \mathrm{x}^{2}+14,17 \mathrm{x}+45,73\left(\mathrm{r}^{2}=0,99\right)^{* *}(\mathrm{PVC} 30)$

$* / * *$ Teste de $\mathrm{F}$ significativo aos níveis de $5 \%$ e $1 \%$, respectivamente.

FIGURA 4 - Curvas e equações de regressão representativas dos valores de pectina solúvel (P.S.) de nêsperas 'Mogi' acondicionadas em filme de PVC de diferentes espessuras e armazenadas a $3 \pm 2{ }^{\circ} \mathrm{C}$ e $85 \pm 3 \%$ UR, por 50 dias.

TABELA 6 - Valores médios de pectina total (P.T.) de nêsperas 'Mogi' acondicionadas em filmes de PVC de diferentes espessuras e armazenadas a $3 \pm 2^{\circ} \mathrm{C}$ e $85 \pm 3 \%$ UR, por 50 dias.

\begin{tabular}{cccccccc}
\hline \multicolumn{7}{c}{${\text { Pectina total }\left(\mathbf{m g . 1 0 0 g}^{-1}\right) \text { no período de armazenamento (dias) }}^{\mathbf{1}}$} \\
\hline Trat.. & $\mathbf{0}$ & $\mathbf{1 0}$ & $\mathbf{2 0}$ & $\mathbf{3 0}$ & $\mathbf{4 0}$ & $\mathbf{5 0}$ & Média \\
\hline CONT. & $563,48 \mathrm{a}$ & $556,89 \mathrm{a}$ & $518,71 \mathrm{a}$ & $548,65 \mathrm{a}$ & $547,47 \mathrm{a}$ & $548,62 \mathrm{a}$ & $547,30 \mathrm{a}$ \\
PVC 15 & $563,48 \mathrm{a}$ & $506,78 \mathrm{~b}$ & $507,97 \mathrm{a}$ & $531,53 \mathrm{ab}$ & $551,87 \mathrm{a}$ & $552,18 \mathrm{a}$ & $535,63 \mathrm{~b}$ \\
PVC 20 & $563,48 \mathrm{a}$ & $541,32 \mathrm{a}$ & $508,74 \mathrm{a}$ & $517,00 \mathrm{~b}$ & $515,73 \mathrm{~b}$ & $489,68 \mathrm{~b}$ & $522,66 \mathrm{c}$ \\
PVC 30 & $563,48 \mathrm{a}$ & $529,87 \mathrm{ab}$ & $507,89 \mathrm{a}$ & $510,66 \mathrm{~b}$ & $506,73 \mathrm{~b}$ & $507,07 \mathrm{~b}$ & $520,95 \mathrm{c}$ \\
\hline
\end{tabular}

${ }^{(1)}$ Médias seguidas de mesma letra na coluna não diferem entre si pelo teste de Tukey a $\mathbf{P}<0,05$.

\section{CONCLUSÕES}

As embalagens com filmes de PVC de espessura 20 e $30 \mu \mathrm{m}$ mostraram-se como as mais adequadas para conservação de nêspera cv. 'Mogi' por um período de até 50 dias a $3 \pm 2^{\circ} \mathrm{C}$ e $85 \pm 3 \%$ UR.
Como os frutos do tratamento PVC 20 $\mu \mathrm{m}$ obtiveram os mesmos resultados que os frutos do tratamento PVC $30 \mu \mathrm{m}$, tem-se que o mais indicado economicamente é o PVC 20 $\mu \mathrm{m}$. 


\section{AGRADECIMENTO}

Ao $\mathrm{CNPq}$, pelo suporte financeiro.

\section{REFERÊNCIAS BIBLIOGRÁFICAS}

BITTER, T.; MUIR, H. M. A modified uronic acid carbazole reaction. Analitical Biochemistry, New York, v. 34, p. 330-334, 1962.

CHACHIN, K.; HAMAUZU, Y. Loquat. In: MITRA, S. (Ed.). Postharvest physiology and storage of tropical and subtropical fruits. Wallingford: CAB International, 1997. p. 397-403.

CHITARRA, M. I. F.; CHITARRA, A. B. Qualidade pós-colheita de frutos e hortaliças: fisiologia e manuseio. Lavras: ESAL/FAEPE, 1990. 543 p.

DING, C. K.; CHACHIN, K.; HAMAUZU, Y.; UEDA, Y.; IMAHORI, Y. Effects of storage temperatures on physiology and quality of loquat fruit. Postharvest Biology and Technology, [S.1.], v. 14, n. 3, p. 309315, 1998a.

DING, C. K.; CHACHIN, K.; UEDA, Y.; MOCHIOKA, R. Changes in polyphenol concentrations and polyphenol oxidase activity of loquat (Eriobotrya japonica Lindl.) fruits in relation to browning. Journal of the Japanese Society for Horticultural Science, Tokyo, v. 67, n. 3, p. 360$366,1998 b$

DING, C. K.; CHACHIN, K.; UEDA, Y.; MOCHIOKA, R. Purification and properties of polyphenol oxidase from loquat fruit. Journal of Agricultural and Food Chemistry, Washington, v. 46, n. 10, p. 4144-49, 1998c.

DING, C. K.; CHACHIN, K.; UEDA, Y.; MOCHIOKA, R. Effect of cold storage and harvest ripeness on the quality and chemical composition of loquat fruits. Food Science Technology International, London, v. 3, n. 2, p. 200-204, 1997a.

DING, C. K.; CHACHIN, K.; UEDA, Y. Effects of polyethylene bag packaging and low-temperature storage on the physical and chemical characteristics of loquat fruits. In: PROCEEDINGS OF THE INTERNATIONAL CONTROLLED ATMOSPHERE RESEARCH CONFERENCE, 7., 1997. Proceedings... [S.1.: s.n.], 1997b. v. 1, p. 177-184.
EL-BULUK, R. E.; BABIKER, E. E.; EL-TINAY, A. H. Biochemical and physical changes in fruits of four guava cultivars during growth and development. Food Chemistry, London, v. 54, p. 279-282, 1995.

FEMENIA, A.; CONESA, M.; SIMAL, S.; ROSSELLÓ, C. Characterization of the cell walls of loquat (Eriobotrya japonica L.) fruit tissues. Carbohydrate Polymers, Barking, v. 35, n. 1/2, p. 169-177, 1998.

FLORES, A. G.; RÍVAS, D. Estúdios de maturación y almacenamiento refrigerado de nispero (Achras sapota L.). Fitotecnia Latinoamericana, Caracas, v. 11, n. 1, p. $43-51,1975$.

HAMAUZU, Y.; CHACHIN, K.; DING, C. K.; KUROOKA, H. Difference in surface color, flesh firmness, physiological activity, and some components of loquat fruits picked at various stages of maturity. Journal of the Japanese Society for Horticultural Science, Tokyo, v. 65, n. 4, p. 859-865, 1997.

INSTITUTO ADOLFO LUTZ. Normas analíticas, métodos químicos e físicos para análise de alimentos. 3. ed. São Paulo, 1985. v. 1, 533 p.

KADER, A. A. Advances in controlled atmosphere applications for quality maintenance of fresh fruits. In: CONGRESSO BRASILEIRO DE FRUTICULTURA, 15., 1998, Poços de Caldas. Anais... Lavras: UFLA, 1998. p. 136-150.

LIMA, L. C. O.; SCALON, S. P. Q.; SANTOS, J. E. S. Qualidade de mangas (Mangifera indica L.) cv. 'Haden' embaladas com filme de PVC durante o armazenamento. Revista Brasileira de Fruticultura, Cruz das Almas, v. 18, n. 1, p. 55-63, 1996.

LOQUAT. Eriobotrya japonica Lindl: fruit facts. 1997. Disponível em: <http://www.crfg.org/pubs/ff/loquat.html>. Acesso em: 26 jul. 2001.

McCREADY, P. M.; McCOMB, E. A. Extraction and determination of total pectic materials in fruits. Analytical Chemistry, Washington, v. 24, n. 12, p. 1586-1588, Dec. 1952.

MOLEYAR, V.; NARASIMHAM, P. Modified atmosphere packaging of vegetables: an appraisal. Journal of Food Science and Technology, Oxford, v. 31, n. 4, p. 267-278, 1994. 
NELSON, N. A. Photometric adaptation of Somogyi method for determination of glucose. Journal Biological Chemistry, Baltimore, v. 153, n. 2, p. 136-175, Jan. 1944.

PENTEADO, S. R. Fruticultura de clima temperado em São Paulo. Campinas: Fundação Cargill, 1986. 173 p.

PÉREZ, A. G.; SANZ, C.; OLÍAS, R.; RÍOS, J. J.; OLÍAS, J. M. Effect of modified atmosphere packaging on strawberry quality during shelf-life. In: INTERNATIONAL CONTROLLED ATMOSPHERE
RESEARCH CONFERENCE, 7., 1997. Davis. Postharvest Horticulture Series, [S.1.], n. 17, p. 153-159, 1997.

VILAS BOAS, E. V. B. Modificações pós-colheita de banana 'Prata' (Musa acuminata x Musa balbisiana grupo AAB) $\boldsymbol{\gamma}$-irradiada. 1995. 73 f. Dissertação (Mestrado em Ciência dos Alimentos) - Universidade Federal de Lavras, Lavras, 1995.

ZAGORY, D.; KADER, A. A. Modified atmosphere packaging of fresh produce. Food Technology, Chicago, v. 42, n. 9, p. 70-77, 1988. 